\title{
ENSEÑAR EN LA UNIVERSIDAD PÚBLICA \\ ARGENTINA: LOS DESAFÍOS DEL \\ OFICIO DOCENTE EN UNA ÉPOCA DE \\ TRANSFORMACIONES
}

\author{
ENSINAR NA UNIVERSIDADE PÚBLICA ARGENTINA: \\ OS DESAFIOS DO DOCENTE EM UMA ÉPOCA DE \\ TRANSFORMAÇÕES
}

\section{TEACHING AT THE PUBLIC UNIVERSITY IN ARGENTINA: CHALLENGES OF TEACHING DURING A PERIOD OF TRANSFORMATION}

\author{
María Paula Pierella ${ }^{1}$ \\ Instituto Rosario de Investigaciones em Ciencias de la Educación (IRICE/CONICET-UNR), \\ Facultad de Humanidades y Artes, Universidad Nacional de Rosario, Argentina
}

\begin{abstract}
Resumen: Este trabajo presenta algunos resultados de un proyecto de investigación centrado en explorar los sentidos acerca del conocimiento y su transmisión, en relatos de profesores/as universitarios/as reconocidos/as por los estudiantes como referentes en su formación de grado en la Universidad Nacional de Rosario, Argentina. Desde un enfoque biográfico-narrativo se abordan las principales transformaciones y desafíos de la docencia universitaria en las últimas décadas, intentando responder una serie de interrogantes transversales al oficio de enseñar: ¿Cuáles son los saberes específicos de un docente universitario?, ¿dónde se adquieren dichos saberes?, ¿qué operaciones se ponen en juego en la transmisión de saberes y experiencias? Se recuperan trayectorias académicas de los docentes que conducen a ampliar la mirada sobre la construcción social del proceso de enseñanza.
\end{abstract}

Palabras clave: Profesores/as universitarios/as. Transmisión del conocimiento. Oficio docente.

\footnotetext{
${ }^{1}$ Doctora en Ciencias Sociales por la Universidad de Buenos Aires; Magister en Educación por la Universidad Nacional de Entre Ríos.
} 
Resumo: Este artigo apresenta alguns resultados de um projeto de pesquisa focado em explorar os sentidos sobre o conhecimento e sua transmissão no relato de professores(as) universitários(as) reconhecidos(as) por estudantes como líderes em sua formação de graduação na Universidade Nacional de Rosário, Argentina. A partir de uma perspectiva biográfica-narrativa, se abordam as principais mudanças e desafios da docência universitária nas últimas décadas, com a intenção de responder a um conjunto de questionamentos transversais ao ofício de ensinar: quais são os saberes especificos de um docente universitário? Onde se adquirem tais saberes? Que operações se colocam em jogo na transmissão de saberes e experiências? Se recuperam trajetórias acadêmicas dos docente que possam ampliar o olhar sobre a construção social do processo de ensino.

Palavras-chave: Professores(as) universitários(as). Transmissão do conhecimento. Oficio docente.

Abstract: This paper shows some results from a research focused on exploring the senses about knowledge and its transmission in university professors' accounts recognized by students as a referent in its training at the Universidad Nacional de Rosario, Argentina. The main transformations and challenges of university teaching in the last decades are tackled from a biographical - narrative approach, trying to answer a series of transversal questions to the teaching profession: Which is the specific knowledge of a university professor?, where is the above mentioned knowledge acquired?, which operations are brought into play in knowledge and experience transmission? Professors 'academic career are retrieved, which leads to expand the perspective on the social construction of the educational process.

Keywords: University professors. Knowledge transmission. Teaching profession.

\section{INTRODUCCIÓN}

En las últimas décadas puede identificarse a nivel global una preocupación cada vez más extendida por el aporte que las universidades nacionales realizan a la llamada "sociedad del conocimiento". Esta expresión, que asigna al saber un lugar central en el futuro de las naciones, alude a un conjunto de transformaciones sociales en las que la información y las nuevas tecnologías que permiten el acceso a ella están en el centro. En ese marco de creciente internacionalización de la educación superior, 
los profesores universitarios se convierten en actores nodales de las diversas propuestas de reforma e innovación que vienen generándose desde fines del siglo XX. Sabido es que el Proceso de Bolonia, que, entre otras cuestiones, pone el eje en el aprendizaje en lugar de la enseñanza, ha concitado extendidos debates sobre el rol docente en estos nuevos contextos. En esos debates, al mismo tiempo que se destaca el valor del conocimiento en las sociedades globalizadas, se reconocen las limitaciones de las instituciones universitarias en el proceso de generación de masa crítica.

En este sentido, si bien en los últimos veinte años tuvo lugar un intenso proceso de masificación de la educación superior, se reconoce que ese ciclo extraordinario entraña otra tendencia estructural clave: las altas tasas de deserción como fenómeno global (EZCURRA, 2011). Y una de las variables que indefectiblemente comienza a ser analizada - además de factores socio-económicos, culturales, institucionales, es la dimensión pedagógica. En este punto la figura del profesor/a univerisitario/a también asume centralidad.

En algunos estudios se señala a la enseñanza como factor de inclusión/exclusión en la universidad (véase entre otros Ezcurra (2011) e Gluz (2011)). Partiendo de los desarrollos de Bourdieu y basándose en trabajos de autores norteamericanos, Ezcurra (2011) afirma que la enseñanza en sentido amplio y en especial las experiencias académicas cotidianas, tienen un papel causal concluyente en la permanencia y en el desempeño académico de los estudiantes.

En trabajos anteriores señalamos que los profesores - especialmente aquellos que se desempeñan en los primeros años- ocupan un lugar destacado en los procesos de afiliación institucional e intelectual de los estudiantes ${ }^{22}$ (PIERELLA, 2014). Si bien, como señala Carli (2012), las tácticas para permanecer en la universidad en ese momento inicial revelan un componente individual importante, ante las limitaciones del dispositivo institucional los profesores son quienes operan a modo de receptores, acechados a su vez por los efectos de la superpoblación en las aulas y las exigencias laborales.

\footnotetext{
${ }^{2}$ Alain Coulon (1997) sostiene que el estatuto de "estudiante universitario" no viene dado por la inscripción en la universidad, sino que implica diferentes temporalidades: el tiempo de la alienación o la extrañeza (entrada a un universo desconocido que rompe con el mundo anterior); el tiempo del aprendizaje (que implica movilización de energías, definición de estrategias y adaptación progresiva); y el tiempo de la afiliación (fase de control y de conversión que le permite al estudiante interpretar - e incluso transgredir - las reglas institucionales). Distingue dos formas de afiliación: la afiliación institucional (comprensión de los dispositivos formales que estructuran la vida universitaria) y la afiliación intelectual o cognitiva (comprensión de lo que se espera de él por parte de los profesores y profesoras y de la institución en general). el proceso de reconocimiento y autorización de profesores y profesoras.
} 
Sobre la base de dichas consideraciones, en este artículo nos interesa aportar algunos elementos para pensar la enseñanza en la universidad, poniendo el foco en profesores que fueron reconocidos por sus estudiantes como figuras claves en sus trayectorias formativas. Al recuperar relatos de los profesores pretendemos abordarlos como actores que, al mismo tiempo que adquieren, producen y transmiten conocimiento, son interpelados para construir reflexividad sobre su propio oficio.

Los avances que aquí presentaremos se nutren de los resultados de un proyecto de investigación posdoctoral, financiado a partir de una beca del Consejo Nacional de Investigaciones Científicas y Técnicas de la República Argentina (CONICET) y titulado La transmisión del conocimiento en la Universidad contemporánea. Un estudio centrado en profesores de dos disciplinas de la Universidad Nacional de Rosario: Física y Letras. Trabajo que se inscribe en el Proyecto CONICET PIP No 1122010010050: Transformaciones de los procesos de adquisición, producción y transmisión del conocimiento universitario. Un estudio de caso centrado en las historias de vida de profesores/as de la Universidad de Buenos Aires del área de humanidades y ciencias sociales, dirigido por la Dra. Sandra Carli en el Instituto de Investigaciones Gino Germani, Facultad de Ciencias Sociales, Universidad de Buenos Aires, República Argentina. A partir de dicho proyecto colectivo se inicia en el mencionado Instituto una línea de investigación centrada en profesores/as que tiene continuidad con proyectos anteriores, también bajo la dirección de la Dra. Carli, abocados al estudio de la experiencia estudiantil en universidades públicas argentinas. En relación con estos últimos, en mi caso, en la tesis doctoral he analizado la construcción de figuras de autoridad en relatos de estudiantes de la Universidad Nacional de Rosario. Las visiones estudiantiles aportaron insumos para pensar aspectos socio-culturales, institucionales, pedagógicos, biográficos, como así también peculiaridades disciplinarias intervinientes en los procesos puestos en juego en la transmisión del conocimiento universitario.

En términos metodológicos la investigación adoptó una estrategia cualitativa sustentada en los supuestos teórico- epistemológicos que dieron lugar a la construcción del problema. Específicamente, el método de investigación es la Teoría Fundamentada (Grounded Theory) de Glaser y Strauss (1967), en la medida en que el proceso de conceptualización procede de los datos obtenidos en la investigación. En esta línea priorizamos la técnica de la entrevista en profundidad con aportes del método biográfico (Sautu, 2004) y la observación no participante.

En relación con lo anterior, se planificaron un conjunto de actividades para la concreción de los objetivos: 
a) a) Entrevistas narrativas a profesores de las carreras de Física y Letras, correspondientes, respectivamente, a la Facultad de Ciencias Exactas, Ingeniería y Agrimensura; y a la Facultad de Humanidades y Artes de la Universidad Nacional de Rosario. En la línea seguida por Bertaux (2005), la entrevista narrativa consiste en la reconstrucción realizada por un sujeto de su vida, o al menos de una parte de ella, a través de una sucesión de hechos y situaciones. Para la obtención de esos datos se cuenta con una guía de preguntas que es flexible y está abierta a las intervenciones de los entrevistados.

b) b) Observaciones no participantes de clases teóricas y prácticas dictadas por los docentes entrevistados con el objeto de revelar las prácticas y discursos presentes en los procesos y prácticas de enseñanza.

La sistematización tiene una doble dimensión: diacrónica-retrospectiva y sincrónica. La primera busca reconstruir los vínculos entre los itinerarios biográficos, las trayectorias y experiencias formativas, académicas y profesionales de los profesores, su posición en el campo universitario y los sentidos construidos en relación con las prácticas de enseñanza. La dimensión sincrónica, por su parte, se orienta a reconstruir las particularidades de las prácticas de enseñanza, situadas en contextos institucionales y áulicos específicos.

La cantidad de observaciones y entrevistas que debían realizarse se definieron por criterios de saturación teórica (GLASER; STRAUSS, 1967) de acuerdo con las dimensiones y problemas que guían la investigación. La técnica de muestreo que utilizamos se desarrolló a partir del muestreo teórico, de Glaser y Strauss (1967). De esta forma, las decisiones iniciales para la recopilación de datos están basadas sobre una perspectiva general del problema y no sobre una estructura teórica preconcebida. Las entrevistas narrativas realizadas fueron diez y se han observado cinco clases.

Siempre siguiendo los parámetros de la Teoría Fundamentada, en el análisis de las entrevistas procedimos siguiendo el método comparativo constante. Es decir, una vez que identificamos categorías recurrentes en una misma entrevista y entre entrevistas diferentes, comenzamos el proceso de codificación o categorización abierta, la confección de memos o elaboraciones preliminares y la determinación de las “categorías núcleo". A partir de allí, y trabajando en un nivel de abstracción mayor, 
establecimos nexos o comparaciones entre las categorizaciones de los entrevistados y las referencias teóricas que consideramos pertinentes.

En este artículo nos centraremos en las entrevistas realizadas a profesores de la Facultad de Ciencias Exactas, Ingeniería y Agrimensura. Si bien los docentes entrevistados refieren a la institución y disciplinas de pertenencia, resultando sus vivencias difícilmente extrapolables, creemos que estas constituyen aportes para pensar la docencia universitaria en términos más amplios.

Los apartados que se presentan a continuación están organizados del siguiente modo: En primer lugar, haremos referencia a las principales transformaciones y desafíos que deben encarar los profesores universitarios argentinos a partir de las últimas décadas, centrándonos principalmente en algunos de los efectos generados por la cristalización de la figura del "docente investigador". Luego, abordaremos una serie de interrogantes claves, según nuestro criterio, para indagar algunos aspectos del oficio de enseñar: ¿Cuáles son los saberes específicos de un docente universitario? ¿Dónde se adquieren dichos saberes? ¿Qué operaciones se ponen en juego en la transmisión de saberes y experiencias? Creemos necesario abordar estas cuestiones no desde orientaciones prescriptivas que apunten a una normalización del trabajo docente desde parámetros impuestos desde afuera, sino a partir de estudios que consideren las voces de los actores involucrados de modo contextualizado. En relación con lo anterior, si el eje de nuestro análisis es el vínculo de los profesores con su propia experiencia en relación con el conocimiento, sólo podemos acceder a ella recuperando insumos de los estudios biográfico-narrativos (DENZIN, 1991; GOODSON, 2004; BERTAUX, 2005; BOLÍVAR; DOMINGO; FERNÁNDEZ CRUZ, 2001, entre otros). Adscribiendo a estas líneas de investigación, advertimos sobre la necesidad epistemológica de conectar las (auto) biografías con el fondo social e histórico en el que se inscriben. En este sentido, el interés principal no radica en el relato de vida en sí mismo sino en la posibilidad de inscribir los datos personales obtenidos a través de las entrevistas en la construcción social del proceso de enseñanza (BOLÍVAR, 2014).

\section{LOS PROFESORES UNIVERSITARIOS FRENTE A LAS TRANSFORMACIONES EN LOS SISTEMAS DE EDUCACIÓN SUPERIOR}

Iniciada la década de 1990 en la Argentina, el “espíritu de época” predominante en los discursos en torno a la Universidad permite vislumbrar el inicio de un nuevo ciclo histórico. En el marco de reformas estatales y administrativas estruc- 
turales y de una adhesión a los lineamientos del llamado Consenso de Washington y su particular visión de la Universidad como un "gasto" que el Estado debía reducir, el vínculo entre gobierno y universidad va a asumir rasgos de mutua desconfianza. Según Naishtat (2004), el contexto universitario argentino, desde la controvertida Ley de Educación Superior N 24521 está signado por la pérdida de confianza en la base; proceso que, leído desde un nivel global, empieza con la reforma británica de los años ochenta y que implica la puesta en escena del Estado evaluador. Concomitantemente, esto traería aparejado un desdibujamiento de una idea clara de la universidad en los propios actores universitarios. En esa misma línea Marquina (2007) señala que el poder de los académicos fue trasladado hacia el Estado y el mercado, con nuevas configuraciones para el trabajo académico en las cuales las características más salientes son la burocratización, la mercantilización y la atomización.

En ese contexto, se observa una tendencia a la sobredimensión de criterios cuantitativos para la evaluación en la carrera académica (CHIROLEU, 2003). Tendencia que en estos últimos años se ha consolidado acompañado por otros procesos como el crecimiento de los posgrados, el incentivo salarial a docentes con doctorado completo, el acceso a subsidios o la dirección de proyectos por criterios de titulación (UNZUÉ, 2011) en tanto orientaciones implementadas a partir de la formulación de políticas de fortalecimiento del sistema de ciencia y técnica (CARLI, 2011; EMILIOZZI, 2011) y el financiamiento externo (GORDON, 2011).

De este modo, se ha ido sedimentando la figura del "docente-investigador", solapándose dos funciones constitutivas de la universidad moderna pero que presentan lógicas y temporalidades diferentes. En efecto, los estudios sobre las formas que adquiere el reconocimiento en el campo científico, coinciden en ubicar a las publicaciones en determinadas revistas internacionales como principal instrumento de consagración y legitimación en un medio cosmopolita (SUASNÁBAR et al., 1997; BECHER, 2001; CHIROLEU, 2003; KNORR CETINA, 2005). Así, la excelencia en la enseñanza cuenta poco para el reconocimiento de los colegas asentados en el mismo campo (BECHER, 2001).

Lejos está de nuestra pretensión intentar dar cuenta de modo exhaustivo de las innumerables variables implicadas en esta tensión entre docencia e investigación. Pero no queremos dejar de advertir la fuerza con que está operando en la dinámica de las instituciones de educación superior en la actualidad.

Dicha problemática ya había sido advertida por Gibbons en la década de 1990, al señalar las dificultades y desafíos que las universidades enfrentarían en el 
futuro, ante el inminente proceso de internacionalización de la educación superior en el marco de la sociedad del conocimiento. Afirmaba este autor:

Paradójicamente, aunque la educación superior se ha desplazado hacia un sistema masivo de enseñanza para un gran número de estudiantes, su orientación fundamental la ha inclinado hacia la investigación. Se considera que el producto de las instituciones de elite es el conocimiento en forma de publicaciones científicas y dispositivos tecnológicos antes que en forma de mentes jóvenes formadas. La mayoría de los docentes, incluso en las instituciones no elitistas, han remodelado correspondientemente sus ambiciones profesionales. (GIBBONS, 1998, p. 17).

De nuestros trabajos anteriores se desprende la percepción negativa que los estudiantes tienen en relación con esta desproporción entre enseñanza e investigación (PIERELLA, 2014). En efecto, uno de los atributos a partir de los cuales se recordaba a los "buenos docentes" fue el hecho de haber manifestado un "compromiso con la enseñanza". Compromiso que muchos de los docentes sostienen que no pueden afrontar como desearían. En la Facultad de Ciencias Exactas, al ser la mayoría de los docentes simultáneamente investigadores del CONICET, encontramos cierto malestar respecto de los criterios de evaluación del sistema científico, entre los que predomina, como señalábamos anteriormente, un criterio cuantitativo en relación con los "papers" publicados en revistas con alto índice de impacto. Frente a esto, muchos de los investigadores expresaron su interés por realizar actividades por fuera de los parámetros de evaluación del sistema de ciencia y técnica. En este sentido, mencionaron experiencias de divulgación científica, experiencias teatrales recuperando algún acontecimiento de la historia de la ciencia, intereses frustrados en realizar investigaciones que guarden relación con la enseñanza de las materias que dictan, etc. Detectamos que aquellos investigadores que ya alcanzaron categorías elevadas como investigadores sienten mayor libertad y seguridad para concretar proyectos de este tipo que aquellos que aún se encuentran en los primeros escalones del sistema. ${ }^{33}$ Desde la perspectiva de Bourdieu (2006) podemos afirmar que la posición en el campo dirige las estrategias y las posibilidades objetivas de los agentes. En este sentido, las estrategias de subversión de algunos investigadores tienen lugar cuando ya se encuentran en una posición relativamente estable dentro del sistema científico.

\footnotetext{
${ }^{3}$ La carrera de Investigador científico y tecnológico del CONICET comprende a las personas que realizan investigaciones en distintos niveles de concepción, diseño, dirección y ejecución. Las categorías que la componen son cinco: Asistente, Adjunto, Independiente, Principal y Superior.
} 
En otras Facultades, como por ejemplo Humanidades, se realizaron críticas al Programa de Incentivos a Docentes Investigadores vigente desde las transformaciones en el sistema universitario encaradas en la década de 1990. Este programa, implementado desde la Secretaría de Políticas Universitarias (SPU) dependiente del Ministerio de Educación de la Nación, instaura un mecanismo de distribución desigual de beneficios en dinero a los docentes que incorpora el criterio de productividad como un modo privilegiado de acumulación de capital simbólico en el campo académico. El objetivo explícito de dicho programa - promover las tareas de investigación y orientar a la planta docente hacia una mayor dedicación a la actividad universitaria - ha sido cumplido sólo en parte. En este sentido, algunos estudios reconocen la combinación de consecuencias esperadas, como el aumento de la producción científica, la tendencia a consolidar grupos de investigación y el mejoramiento salarial, con efectos no deseados, como situaciones de apariencia de investigación, exceso de competitividad entre colegas y falta de estabilidad en los incrementos salariales (BUCHBINDER; MARQUINA, 2008). En efecto, muchos de los entrevistados señalaron que el programa de incentivos habría condicionado la proliferación de investigaciones de baja calidad o de "simulacros de investigación" que generarían ciertas tensiones en las comunidades académicas.

\section{PENSAR AL DOCENTE UNIVERSITARIO DESDE SU OFICIO}

Como señalamos en el apartado anterior, se estaría evidenciando un desequilibrio, a favor de la primera entre las funciones de investigación y enseñanza. Y esto es problemático, porque redunda en una drástica disminución del tiempo dedicado por los docentes a pensar temas específicos de la enseñanza en el grado y que guardan relación, entre tantas otras cuestiones con la selección de temas y problemas a trabajar, la organización de la clase, la reflexión sobre las formas más apropiadas de enseñar cada tema, las estrategias más adecuadas a encarar según el grupo en cuestión, etc.

Considerando la complejidad de esta situación es que creemos necesario promover procesos de reflexividad que tiendan a recuperar la especificidad de los saberes sobre la enseñanza en la universidad. Saberes que, en la gran mayoría de los casos, permanecen en estado tácito y que, desde enfoques biográfico-narrativos podemos contribuir a visibilizar. En muchas ocasiones, aquellos profesores cuyas prácticas producen efectos positivos en las trayectorias académicas de los estudiantes, no disponen de un saber pedagógico formalizado que pueda traducirse bajo la forma de 
un "saber hacer". En relación con esto, creemos que la narración de las experiencias puede potenciar una reflexión sobre la construcción social del proceso de enseñanza en clave de oficio. Es decir, en clave de preguntas relacionadas con los modos de encarar el trabajo. ${ }^{44}$

\subsection{ACERCA DE LOS SABERES INVOLUCRADOS EN LA ENSEÑANZA UNIVERSITARIA}

Cualquier oficio, profesión, trabajo se sostiene, fundamenta y legitima sobre la base de algún tipo de saber que lo diferencia de otro. ¿Cuál es la especificidad de los saberes de los docentes universitarios? Lejos de buscar una clasificación a priori de saberes a modo de tipología que no conduce muy lejos, pretendemos articular una mirada socio-histórica de la disciplina con los relatos de estudiantes y docentes, de modo tal de identificar tradiciones arraigadas, espacios vacantes y desafíos que se nos presentan a quienes intentamos pensar la pedagogía universitaria.

La perspectiva de los estudiantes nos llevó a afirmar que el "saber erudito" sobre la propia disciplina es un aspecto clave. Saber sobre la materia que se enseña, investigar sobre el propio objeto de conocimiento, sostener una actitud apasionada en torno a las problemáticas disciplinares generaría ante los estudiantes la imagen de alguien cuyo recorrido académico lo autoriza para hablar de determinado tema, para intervenir en los debates propios de determinada área del conocimiento. Generalmente cuando los estudiantes hablan de los buenos profesores dicen que saben mucho de su materia y que transmiten una pasión por el conocimiento que enseñan. Sin embargo, señalan algo más, y es que un buen profesor "sabe enseñar". Esto se evidenciaría en el modo en que presenta la materia, la predisposición para explicar, para responder preguntas, el modo de armar una evaluación, etc. Así, la clave disciplinar se complementa con la clave pedagógica. Contenido y forma quedan de este modo tan articulados que resulta imposible discernir la preeminencia de uno de los dos. En otras palabras, los saberes sobre la forma no pueden pensarse de forma aislada, por fuera del contenido a enseñar. Clark (1983, p. 53) sostenía que ante la pregunta ¿qué es la educación superior?, “[...] aunque el verbo aparece insistentemente en singular, la respuesta debe ser plural e indirecta." En consecuencia, “[...] a partir de la sustancia, se derivan ciertos aspectos de la forma." (CLARK, 1983, p. 40). Así, en torno a ciertas tareas intelectuales específicas, las disciplinas - entendidas como las "empresas"

${ }^{4}$ Para profundizar la noción de oficio docente recomendamos consultar, entre otros Dubet (2006) y Antelo y Alliaud (2009). 
primordiales de los sistemas académicos que operan con sus propios procedimientos modelados a través del tiempo - operan con una determinada tradición cognitiva, con sus categorías de pensamiento y códigos de comportamiento que hacen a su cultura (CLARK, 1983).

Es preciso aclarar que cuando hablamos de las formas que asume la transmisión de conocimientos no podemos dejar de considerar el peso que tiene la personalidad de quien transmite. Como ya destacaba Freud, evocando sus años escolares, ambas cuestiones resultan difícilmente escindibles. ${ }^{55}$

Ahora bien, la tradición de la docencia universitaria, en general, ha desestimado al saber pedagógico, relegándolo a otros niveles del sistema educativo. Desde ciertas miradas emblemáticas, para enseñar en la universidad el saber sobre el propio objeto de conocimiento se complementaría con cierto talento individual. En relación con lo anterior, diferentes pensadores emblemáticos en el campo de las ciencias sociales se han pronunciado sobre esta cuestión a lo largo de todo el siglo XX.

En 1919, en una conferencia titulada "La ciencia como vocación", ante la Asociación libre de estudiantes de Munich, Max Weber afirmaba que todo joven que se crea llamado a la profesión académica debe ser consciente de la doble vertiente de la tarea que le aguarda. "No le bastará con estar cualificado como sabio, sino que ha de estarlo también como profesor y estas dos cualidades no se implican recíprocamente ni muchísimo menos. Una persona puede ser un sabio excepcional y al mismo tiempo un profesor desastroso.” (WEBER, 1972, p. 188). Según el sociólogo, exponer los problemas científicos de modo que resulten comprensibles y lograr que las mentes "no educadas" pero "capaces" tengan sobre ellos ideas propias, es para él la más difícil de las tareas pedagógicas. Pero el arte de enseñar es para Weber un don personal que nada tiene que ver con la calidad científica de un sabio.

Varias décadas después - en 1965 - y desde otra perspectiva, en una conferencia pronunciada en el Instituto de Investigación Docente de Berlín titulada “Tabúes relativos a la profesión de enseñar", Adorno (2003, p. 9) planteaba precisamente que los docentes que en Alemania gozaban de mayor reputación, los profesores universitarios, “[...] no se fijan en el ámbito pedagógico, sospechado de ilusorio.” Estas apreciaciones tienen sentido en las disquisiciones del filósofo alemán en la medida en

\footnotetext{
${ }^{5}$ Leemos en Sobre la psicología del colegial "La emoción experimentada al encontrarme con mi antiguo profesor del colegio me conmina a una primera confesión: no sé qué nos embargó más y qué fue más importante para nosotros: si la labor con las ciencias que nos exponían o la preocupación con las personalidades de nuestros profesores. En todo caso, con éstos nos unía una corriente subterránea jamás interrumpida, y en muchos de nosotros el camino a la ciencia sólo pudo pasar por las personas de los profesores: muchos quedaron detenidos en este camino y a unos pocos - ¿por qué no confesarlo?- se les cerró así para siempre." (FREUD, 1996, p. 1893).
} 
que este señala una diferencia pertinente entre el estudio académico y el aprendizaje escolar. Según el autor, en la universidad debería disminuir el interés por que todo se complete paso por paso, sin lagunas. Dedicándole tiempo al estudio de una materia, a través de la reflexión en torno a esta, sucedería algo así como un salto cualitativo por el cual se iluminan ciertas cuestiones que antes no eran evidentes (ADORNO, 2002).

Durante los mismos años pero desde una mirada crítica hacia la deslegitimación del saber pedagógico en la Academia francesa, Bourdieu y Passeron (2003) señalaban en Los herederos que todo esfuerzo por introducir una disciplina "educativa" en la enseñanza superior era percibido por los estudiantes y los profesores como atentatorio contra la dignidad de unos o como incompatible con la maestría de otros. Desde su perspectiva, la adhesión a la ideología carismática del talento o la gracia individuales habrían tendido a ocultar el peso de la herencia social o del trabajo disciplinado en las prácticas de enseñanza. Los autores partían de un personaje que en los años sesenta constituía el tipo ideal de estudiante por su carácter dominante en términos cuantitativos y cualitativos: la del heredero. Figura estudiantil de una universidad de origen social burgués, el heredero expresaba el peso de las determinaciones sociales en la posibilidad de ingresar a la universidad y personificaba la integración de un estilo intelectual y un estilo de vida caracterizados, entre otras cosas, por un desinterés respecto de las técnicas pedagógicas - propias de los rituales escolares - que atentarían contra la imagen romántica del trabajo intelectual como actividad libre e inspirada (BOURDIEU; PASSERON, 2003, p. 95).

Ahora bien, las argumentaciones anteriores, avalando o discutiendo la necesidad de tener en cuenta los saberes pedagógicos en la formación de docentes universitarios, coinciden en que cuando refieren a "lo pedagógico" están pensando en cuestiones "técnicas". De modo contrario, desde las perspectivas que nos orientan planteamos que la transmisión de conocimiento no es un acto mecánico, no es una operación meramente "técnica", sino que es un proceso de significación. Transmitir "algo" es un proceso siempre abierto a la reformulación del destinatario e implica atender a las singularidades de este proceso. En la medida en que la transmisión es una relación dialógica, marcar el "yo" desde el cuál se habla y reconocer al otro como sujeto son operaciones centrales (CORNU, 2004).

Vale preguntarse, entonces ¿Qué suscitan en nosotros los temas, autores, enfoques, que buscamos transmitir? ¿Qué lugar tiene en la clase la explicitación de un recorrido, el relato de las propias aventuras en relación con el conocimiento? ¿En qué posición se pone al otro? ¿Cuáles son los imaginarios y expectativas en relación con las nuevas generaciones que ingresan a la universidad? ¿Qué porciones del saber acu- 
mulado se pretende transmitirles? ¿Cómo? Interrogantes que exceden una reflexión sobre las técnicas para abarcar cuestiones políticas, culturales y éticas.

\section{2 ¿CÓMO Y DÓNDE SE ADQUIEREN DICHOS SABERES?}

En las trayectorias académicas de los docentes entrevistados no se registra el pasaje por instituciones de formación docente, pero sí una serie de vivencias personales, escolares y profesionales. Estas adquieren relevancia en los relatos como aspectos que, en el presente, y retroactivamente - como señalábamos en el apartado anterior - tuvieron algo que ver en su sabiduría "práctica". Nos encontramos con docentes expertos que pueden dar sentido a su trabajo, que ponen en funcionamiento el pensamiento en el proceso mismo de producción de sus prácticas. Docentes apasionados por lo que hacen, inquietos por encontrar diferentes formas de transmitir un contenido, comprometidos con su tarea. No olvidemos que nuestros entrevistados son profesores reconocidos por sus estudiantes como "buenos enseñantes" y que todas esas cuestiones fueron señaladas por los jóvenes como factores que les habrían facilitado el pasaje por la universidad. Y sin embargo, la preocupación por "enseñar mejor" aparece de modo reiterado. E incluso muchos de ellos se sorprenden cuando escuchan que sus alumnos los han reconocido con admiración. El hecho de no tener conocimiento sobre el producto de su obra generaría cierta inquietud. En función de lo anterior podemos presumir, siguiendo a Antelo (2009), que sólo sabemos que hubo una enseñanza cuando hay aprendizaje, y no antes. Y en la mayoría de los casos, esto último pasa desapercibido para el propio docente. El problema, insiste Antelo (2009, p. 15-16), quizás radique en lo siguiente:

[...] aparentemente, el saber que uno produce acerca de la enseñanza es un saber que siempre llega después de los hechos, a posteriori. No es que no haya un saber enseñar, un cómo enseñar mejor. Pero siempre uno accede a ello después de los hechos [...] Suscitar el deseo de aprender. Despertar la curiosidad. Sí, claro. Pero ¿cómo sabemos que esto sucede? Tarde, siempre cuando ya ha sucedido. Y no sólo tarde, sino siempre dejándonos guiar por la dubitativa veracidad del relato y por el tiempo verbal que no deja mentir. ${ }^{66}$

De este modo, siempre a posteriori y seguramente con las traiciones que la memoria ejerce, al relatarlo, sobre el pasado, los docentes de nuestro trabajo recuperaron algunas de las "marcas" que otras enseñanzas dejaron en ellos. Y hablaron en-

${ }^{6}$ Las cursivas pertenecen al autor. 
tonces de lugares y figuras de formación. A continuación nos detendremos en algunos de ellos.

\section{-La experiencia:}

Los profesores entrevistados cuentan con una trayectoria relativamente larga - veinte años como mínimo- en docencia universitaria. Durante todo ese tiempo hay saberes en relación con la propia práctica que se han ido sedimentando, principalmente por efecto de probar, ensayar, reconocer errores y volver a probar. En este sentido, la experiencia es reconocida como un proceso de aprendizaje a partir del cual pudieron elaborar recursos propios que utilizan en diferentes situaciones. Muchos de esos "recursos experienciales", tomando la expresión de Delory-Momberger (2014), se encuentran en estado tácito, pre-reflexivo.

En su libro El artesano, Sennett (2009, p. 68-69) sostiene que el saber tácito se alcanza en las esferas más elevadas del pensamiento:

Los expertos pueden dar por supuesto una serie de operaciones que llevan todo un proceso de adquisición. Para lograr la asimilación de un contenido: conversión de información y práctica en un conocimiento tácito. En las fases superiores de la habilidad, hay una constante interrelación entre el conocimiento tácito y el reflexivo; el primero de los cuales sirve como ancla, mientras que el otro cumple una función crítica y correctiva.

Este proceso involucra esquemas de pensamiento y decisión de los que depende la puesta en práctica de los saberes en una situación compleja (PERRENOUD, 1994).

Ahora bien, en los relatos pudimos identificar ciertos matices a la hora de pensar la experiencia. Es decir, si bien el recorrido transitado, las pruebas realizadas, generan cierta tranquilidad y seguridad, también esto puede llevar a situaciones de comodidad que irían en detrimento de una buena enseñanza. Decía un profesor de la carrera de Física:

Esta materia hace mucho que la doy, no sé si me cansa pero uno es como que se descuida y no tengo que estudiar nada, el día anterior miro algo [...] Y eso lleva a que uno la de "de taquito", y da cosas que al principio te costaba entender y despúes las entendés tanto que lo das sobreentendido para el alumno ${ }^{77}[\ldots]$

\footnotetext{
${ }^{7}$ La expresión "de taquito" se usa coloquialmente para referir a algo que se logra hacer bien fácilmente, sin demasiado esfuerzo.
} 
Cuando uno da la materia mucho tiempo, a más años que das es menor el tiempo que le dedicás fuera del pizarrón. Salvo las consultas, el tiempo de sentarse, de leer y preparar es menor porque ya tenés todo listo $[\ldots]^{88}$

Vemos aquí que esos conocimientos tácitos apropiados por fuerza de la experiencia pueden también resultar un obstáculo en materia de enseñanza. En la medida en que pueden llegar a dificultar ciertos procesos de empatía con los estudiantes. Empatía que implicaría, entre otras cuestiones, desmontar paso a paso ese conocimiento que ha quedado integrado en una rutina (SENNETT, 2009). Desde esta perspectiva, retornar a la inseguridad propia del novato - y la expresión vale aquí tanto para el docente como para el estudiante - puede ayudar a orientar anticipando las dificultades, llamando la atención sobre puntos conflictivos, subrayando cuestiones de importancia. A su vez, la sedimentación de lo ya probado, puede cerrar el paso a nuevos procesos de experimentación.

\section{-La cátedra:}

La cátedra ha sido la forma tradicional de control operativo de las tareas docentes en la mayor parte de las universidades europeas y latinoamericanas, remontándose a la universidad medieval organizada en torno a una jerarquía interna basada en el modelo gremial (CLARK, 1983). Aun en la actualidad, estas últimas constituyen uno de los ámbitos más significativos en las trayectorias de formación de los profesores universitarios. Para muchos de ellos las cátedras brindarían una formación de tipo artesanal, sostenida en el vínculo maestro-discípulo, donde el primero guía y controla el proceso de trabajo y de formación, y el segundo va adquiriendo los secretos del oficio a partir de realizar determinadas tareas con la orientación del superior (ANIJOVICH, 2013). Sin embargo, el sentimiento de haber sido acompañados por el superior a la hora de formarse como docentes suele ser una excepción. Uno de nuestros entrevistados lo relataba de esta manera:

Peter y otro profesor iban a mis clases cuando yo era auxiliar y lo importante es que me hacían comentarios sinceros, que es muy raro eso en Física. Te dan el cargo y nunca más nadie te da un consejo de cómo dar clases; salvo que te encuentres con esta gente...Para muchos sentirse observados es visto como algo

\footnotetext{
${ }^{8}$ Entrevista realizada el 16 de julio de 2012 en el Instituto Rosario de Investigaciones en Ciencias de la Educación (IRICE-CONICET/UNR).
} 
negativo...Yo la primera clase explico cuál es el sentido de la materia, por qué se da lo que se da, y eso lo tomé de Peter $[\ldots]^{99}$

En algunos casos se hizo referencia a la cátedra como un lugar en el que la tarea se realiza de un modo un tanto mecánico o estructurado, sin reflexiones a posteriori. El titular brindaría instrucciones operativas que regulan el oficio y ordenan los procedimientos. Lo cual redundaría en sentimientos de mayor seguridad:

En una estructura de cátedra, de abajo para arriba, se mandan instrucciones...El profesor titular hacía los cronogramas, cúando se daba tal tema, qué material se usaba...Eso te facilitaba las cosas, porque vos no decidís nada...Es como que se respetaba lo que decían los papeles: El JTP tiene que hacer tal cosa, el auxiliar tal otra $[\ldots]^{10}$

No obstante, suele suceder que muchos profesores de gran trayectoria como investigadores y años de experiencia en la docencia perciban su posición en la cátedra como una situación de subordinación que afectaría su autonomía para trabajar. En esos casos, se valoran los modos de ejercicio del poder en el cual las relaciones asumen un carácter más simétrico:

Hay algunos profesores que se están jubilando, afortunadamente, que tienen una idea del "profesor", o sea del profesor que iba una hora, tiraba un lineamiento, y después los JTP o ayudantes hacían el trabajo pesado, o los alumnos iban y estudiaban... Pero ya casi no quedan esos casos. De hecho hay materias donde no hay tanta distinción entre los docentes que están a cargo. La distinción se da por una cuestión generacional, cuando hay un profesor que da la teoría y tiene sesenta y pico de años y el ayudante tiene veinte, treinta, por ahí $[\ldots]^{11}$

Así, observamos que, desde el punto de vista de la división del trabajo, las relaciones jerárquicas propias de la cátedra al mismo tiempo que brindarían cierta seguridad, suelen generar cierto malestar y fragmentación entre sus miembros.

Es digno de considerar que muchos de los docentes que conformaron nuestro corpus de entrevistados trabajan al mismo tiempo en las carreras de Ingeniería y

\footnotetext{
${ }^{9}$ Entrevista realizada el 16 de julio de 2012 en el Instituto Rosario de Investigaciones en Ciencias de la Educación (IRICE-CONICET).

${ }^{10}$ Entrevista realizada el 18 de julio de 2012 en la Facultad de Ciencias Exactas, Ingeniería y Agrimensura de la Universidad Nacional de Rosario.

${ }^{11}$ Entrevista realizada el 22 de abril de 2013 en el Instituto de Mecánica aplicada y Estructuras (IMAE) de la Facultad de de Ciencias Exactas, Ingeniería y Agrimensura de la Universidad Nacional de Rosario.
} 
Física, la primera organizada a partir de cátedras y la segunda por departamentos. Esto los llevaría a establecer comparaciones entre unidades operativas que concentran las responsabilidades en la persona del titular y organizaciones, como en el caso de los departamentos, en los que las responsabilidades y el poder se distribuyen entre varios profesores de rango superior semejante y se admite mayor participación de las demás categorías (CLARK, 1983).

\section{-Figuras memorables:}

Utilizamos aquí la noción de figuras inspirados por la idea de "retazos discursivos" de Barthes (1982). Es decir, no como un modelo unívoco que ejerce influencia directa sobre los sujetos, sino como elementos heterogéneos que confluyen en el armado - personal, si se quiere - de un estilo de docencia.

Como cuestión general, todos los profesores entrevistados reconocen en sí mismos las marcas de algún profesor emblemático de quien habrían tomado algunos rasgos visibles en sus prácticas. En palabras de Jackson (2002, p. 33-34), se pusieron de manifiesto una serie de evocaciones que "[...] forman una enciclopedia internalizada de información pedagógica, aportada por los docentes que han conocido en el pasado." Uno de los docentes recurría a su propia historia formativa para explicitar su interés por alentar en los estudiantes las preguntas, la curiosidad que los movilizó a iniciar la carrera de Física:

- Durante la primaria, tuve experiencias muy negativas en relación a los docentes. Siempre recuerdo que la maestra me puso en ridículo frente al aula por decir mis ideas. Y no eran mis ideas, lo había leído en un libro sobre que el aire tiene peso y es una presión que se ejerce sobre nosotros. Lo dije en la primaria, me acuerdo, y la maestra hizo llamar a todos la atención para que rieran, básicamente [...] Sin embargo yo estaba convencido porque lo había leído en libros, había curioseado en libros. En esa época no había nada de Internet. Pero después en la secundaria tuvimos un profesor que era docente en la facultad, por esas cuestiones de la época, era docente en la Universidad de Entre Ríos, que se había cerrado por el Proceso. Y él trasladó todo su laboratorio o todos sus instrumentos de enseñanza que tenía en la universidad, las trasladó al colegio. Entonces las clases realmente eran clases abiertas en el laboratorio de Física. Y la manera en que nos planteaba las preguntas y cómo nos motivaba a la curiosidad fue para mí determinante. Fue reencontrar ese placer que de alguna manera tenía de niño. Reencontrarme con esa manera de acceder a la pregunta y a la búsqueda de la 
respuesta. Estoy convencido de que sentí muchas ganas ahí de empezar a estudiar Física $[\ldots]^{12}$

El peso de las propias biografías escolares, las marcas de quienes fueran sus docentes se pone en evidencia aquí mostrando la multiplicidad de internalizaciones, frustraciones, identificaciones, que operan en el trayecto formativo. Asimismo, en el fragmento anterior podemos observar la relación entre determinadas experiencias biográficas y formativas y los sucesos históricos. El hecho de que un profesor de la secundaria haya encontrado en este nivel del sistema un espacio en el cual poner en funcionamiento aquello que en la universidad estaba vedado fue crucial, desde la perspectiva del entrevistado, en su trayectoria académica. Lo anterior nos lleva a apuntalar la idea de que no existe saber - saber pedagógico, en este caso- que no esté inscripto en relaciones de saber y que no esté sometido a procesos de capitalización y transmisión (CHARLOT, 2008). Esto tendería a relativizar cierta idea de que la predisposición para transmitir sería una cuestión absolutamente personal.

Ese juego complejo de relaciones involucra figuras de referencia no sólo reductibles a personas. También ocuparon un lugar destacado diferentes producciones culturales indirectamente relacionadas con el oficio docente que actuaron a modo de aprendizajes implícitos. Así, se mencionó al teatro como ámbito de aprendizaje de actitudes y formas de representación que favorecerían una buena enseñanza, la literatura como un discurso que permite trascender el saber disciplinar y abrir el conocimiento hacia nuevos horizontes, prácticas de yoga como un camino hacia la meditación que impactaría en la enseñanza, experiencias de divulgación de la ciencia como un modo de ejercitar la transposición de contenidos de gran complejidad.

Desde la perspectiva del filósofo español Pardo (2004), podríamos encontrar en esos ámbitos ciertas reglas implícitas de acciones, o en todo caso ejemplos, que se presentan como anteriores a la comprensión teórica, pero cuyo carácter concreto las dotaría de gran eficacia.

\subsection{LA TRANSMISIÓN DE SABERES Y EXPERIENCIAS}

Como señalábamos al comienzo de este artículo, sobre la universidad recaen una serie de diagnósticos sobre lo problemático que resulta el proceso de transmisión de los saberes eruditos, científicos, profesionales, etc. "Declive de la institu-

\footnotetext{
${ }^{12}$ Entrevista realizada el 22 de abril de 2013 en el Instituto de Mecánica aplicada y Estructuras (IMAE) de la Facultad de de Ciencias Exactas, Ingeniería y Agrimensura de la Universidad Nacional de Rosario.
} 
ción” (DUBET, 2006), “crisis de esa buena idea” (NAISHTAT; GARCÍA RAGGIO; VILLAVICENCIO, 2001), podríamos seguir recuperando enunciados que dan cuenta de las dificultades de diversa índole que sufre la institución desde un punto de vista social e institucional, pero también pedagógico.

Desde la perspectiva de los docentes, una de las dificultades más importantes es encontrar modos de transmitir conocimientos que favorezcan el aprendizaje de los estudiantes. Y este simple enunciado que remite a la relación entre enseñanza y aprendizaje, cuya complejidad y no causalidad ha sido ampliamente estudiada por la pedagogía, no deja de presentar una cantidad importante de aristas: ¿Enseñar bien qué cosa? ¿Enseñar bien a quiénes? ¿Enseñar bien para qué? Vayamos por parte.

Por un lado, la preocupación de los docentes remite al objeto de conocimiento: “¿Cómo enseñar mejor determinado contenido?” “Cómo mejorar la transmisión de conceptos a los estudiantes?", “Cómo generar experimentos que nos permitan acercar al alumno a determinados conceptos?" Interrogantes, formulados en las entrevistas, que se relacionan directamente con las tensiones entre el saber y su explicación, o, de modo más general, entre el lenguaje y la acción.

Sennett (2009), comparando los laboratorios científicos con talleres de artesanos, se interna en estas dificultades que se presentan cuando alguien quiere explicar a los demás una técnica o un procedimiento. Todos vivimos eso cuando pretendemos seguir las instrucciones para armar algo, o en una receta de cocina. En el taller o el laboratorio, o en una clase, la palabra hablada que acompaña una demostración parece más eficaz que las instrucciones escritas, porque allí tendríamos la retroalimentación del otro a través de su mirada, de sus preguntas e intervenciones. También suele suceder, como se pone de manifiesto en la siguiente cita, que uno mismo obtenga aprendizajes en este proceso, que el docente mismo se enriquezca al realizar, por ejemplo, un experimento:

El hecho de poder hacer las cosas uno en el laboratorio, de verlas y experimentarlas uno, tiene un valor para mí. Creo que hay como dos instancias. Una, uno lee un libro de física y conceptualmente cree que entendió las cosas. Cuando hace los ejercicios prácticos propuestos por el libro, ahí hace una primera confrontación de si entendió. Y la segunda es cuando vos querés hacer el experimento, que hay como un salto. Porque uno puede llegar a responder ejercicios porque entendió el concepto, incluso puede resolverlos bien. Pero hay otro paso más cuando uno trata de repetir el experimento, de decir bueno, cómo se llegó a esta conclusión. Haciendo esto, poniendo esto, pero si yo cambio acá [...] Y esas alternancias generan nuevas preguntas, que hacen que se enriquezca el concepto aprendido, 
o que se cuestione. Uno empieza a dudar si lo aprendió bien. Entonces hay algo interesante en esas materias, que son prácticas, que son concretamente hacer un experimento. ${ }^{13}$

Ahora bien, también tenemos que tener en cuenta aquí que el diálogo puede ser confuso. Creemos que podemos reemplazar la explicación por la demostración, pero el aprendizaje por demostración da por supuesto la posibilidad de la imitación directa, dice el sociólogo. Es cierto que a menudo funciona bien, pero con la misma frecuencia fracasa. Muchas veces el profesor no es capaz de mostrar el error sino sólo la manera de hacer algo bien, le resulta difícil retroceder a la situación elemental del alumno (SENNETT, 2009). El desafío, dice Sennett, es lograr que las instrucciones transmitan información, está en crear “instrucciones expresivas”. Y así, basándose en el arte de la cocina presenta una serie de ejemplos que favorecerían la explicación, tales como:

a) Ilustración empática: intentar ponerse en el lugar del otro anticipando puntos problemáticos, advirtiendo sobre errores frecuentes, retrocediendo hasta la situación inicial en que se encuentra el estudiante.

b) Presentación del escenario: contar historias relacionadas con el tema, evocar el contexto cultural, utilizar datos secundarios trayendo a colación hechos, anécdotas, observaciones.

c) Utilización de metáforas, ejemplos, analogías: enriquecer el lenguaje de modo tal de cargar de peso simbólico a la acción.

Todas estas constituyen instrucciones invalorables a ser consideradas a la hora de enseñar conceptos, categorías, principios, operaciones, problemáticas, como así también a la hora de "enseñar a enseñar". Y, sin embargo, como afirma Serra (2010, p. 79),

[...] un conjunto de instrucciones a seguir no hacen a un buen docente, $o$, para decirlo de otro modo, la enseñanza no se reduce a un conjunto de instrucciones bien cumplidas. El que enseña pone algo que excede a las reglas del buen enseñar: voluntad, según algunos, pasión según otros, obstinación, deseo, etc.: todos términos irreductibles a una regla o una instrucción.

\footnotetext{
${ }^{13}$ Entrevista realizada el 22 de abril de 2013 en el IMAE.
} 
En segundo lugar, cuando se habla de las dificultades para enseñar determinados saberes, indefectiblemente aparecen problemáticas en relación con el otro, o con los otros a quienes se enseña. Durante las últimas décadas, los procesos de masificación de la educación superior implicaron transformaciones en el perfil del estudiante universitario que tradicionalmente asistía a la universidad. El crecimiento del sistema universitario público se combina con el acceso de jóvenes pertenecientes a sectores sociales cuyo capital cultural no suele ser el esperado en las instituciones universitarias. Frente a esto, es frecuente escuchar expresiones que enfatizan los "déficits": "La escuela secundaria no los formó bien", "no les dio los contenidos necesarios para ingresar a la universidad", "no disponen de disposiciones familiares y sociales apropiadas para encarar una educación superior", etc. etc. Es decir, cuesta considerar a todos los estudiantes como sujetos de derecho y modificar prácticas y representaciones en función de dicha consideración (RINESI, 2013).

Por último, tienen lugar nuevas tensiones, que no podemos pensar por fuera de las dos anteriores, que se generan al pensar ya no en cómo transmitir mejor un contenido a estudiantes determinados, sino en cómo ofrecer saberes que sean útiles para resolver problemas o situaciones de la práctica. Perrenoud (1994) afirmaba hace veinte años la ausencia de una reflexión sobre estos saberes en el marco de la universidad. Sostenía que esta institución podía elaborar un plan de formación extremadamente explícito en lo que hace a los saberes de referencia (disciplinarios, didácticos, pedagógicos, sociológicos), mientras que los saberes prácticos quedaban en un “agujero". En la actualidad podemos observar, de modo contrario, una proliferación del lenguaje de las “competencias". Desde ciertas perspectivas, pareciera ser que los planes de estudios de todas las carreras debieran reformularse de modo tal de poner el centro en las competencias que el medio requiere de ese profesional. Ahora bien, esas "competencias" o habilidades no pueden pensarse de modo aislado, por fuera de las relaciones - entre estudiantes, profesores y saberes-que se van generando en cada situación determinada. Los estudiantes recuerdan en general con admiración a aquellos profesores que se preocupan por transmitir anécdotas, secretos, particularidades, ejemplos de su experiencia profesional, es decir, algunos "gajes del oficio". Nuevamente, lo que aparece aquí, es el reconocimiento de la necesidad de contar con referentes "con oficio" que orienten el proceso formativo (ALLIAUD; ANTELO, 2009). Y decimos oficio y no competencias. Hacemos referencia al oficio concebido como capacidad de producir algo, de conocerlo, de hacerlo reconocer (DUBET, 2006).

Desde la perspectiva que adoptamos en este trabajo, los saberes que se transmiten en la universidad no pueden pensarse por fuera de una reflexión sobre 
las formas en que se produce dicha transmisión. Parafraseando a Alliaud y Antelo (2009), creemos que los estudiantes tendrían más chance de operar sobre situaciones complejas, si gente con oficio y preocupada por él los prepara para ello. Enseñar el oficio, y sobre todo cuando pensamos en el oficio docente, es practicarlo. "Y practicarlo mucho. En este sentido, los resultados o buenos resultados se pueden relacionar con prácticas sostenidas y coherentes con lo que se intenta transmitir." (ALLIAUD; ANTELO, 2009, p. 94).

\section{CONSIDERACIONES FINALES}

En este trabajo, basándonos en una investigación sobre profesores reconocidos por los estudiantes como referentes en su proceso de formación en la Universidad Nacional de Rosario, pretendimos aportar elementos para pensar algunas problemáticas que atraviesan a la docencia universitaria en la actualidad.

Paradójicamente, en un contexto en el que la función de enseñanza se encuentra debilitada, por el énfasis puesto en la figura del “docente-investigador", los discursos que sostienen la necesidad de incrementar en los docentes universitarios el saber sobre la enseñanza se ponen de manifiesto en la creciente creación de especializaciones y posgrados de índole pedagógico-didáctica. En otras palabras, la tradición disciplinar propia de la docencia universitaria - que asimilaba saber pedagógico con técnicas de enseñanza- se vería cuestionada, al mismo tiempo que la enseñanza se subordina a la investigación en las representaciones de diferentes actores universitarios.

Afirmando que la enseñanza es una variable clave en el proceso de afiliación de los estudiantes a la institución, quisimos darle visibilidad al oficio docente, entendiendo a este como la capacidad de conocer y producir obras de enseñanza que se puedan reconocer. Así, ¿cuáles son los saberes específicos de un docente universitario? ¿Dónde se adquieren dichos saberes? ¿Qué operaciones se ponen en juego en la transmisión de saberes y experiencias?, fueron los interrogantes que orientaron nuestro recorrido.

En relación con el primer interrogante, nuestra investigación nos permite sostener que la docencia universitaria, en la medida en que está involucrada en procesos de producción y transmisión del conocimiento, requiere por parte de los profesionales un trabajo que puede pensarse en torno a dos claves principales. Por un lado, el trabajo académico en relación con la propia disciplina. Esto implica una actitud investigativa constante, de interrogación sobre el conocimiento, sobre el objeto respecto del cual el profesional se especializa. Pero por otro lado tiene que pensar ese 
conocimiento y el ejercicio de la profesión en clave pedagógica, es decir, en clave de transmisión. Porque la enseñanza universitaria plantea una relación entre el profesor, que se va especializando en determinado tema, un contenido disciplinar y los estudiantes. Esto requiere pensar profundamente la relación entre el contenido y la forma. Entre qué se va a transmitir y cómo se lo hace. Lo cual comprende cuestiones técnicas - o metodológicas- pero va más allá de esto. Implica una reflexión sobre la selección de los contenidos, sobre el recorte que se hace, sobre la organización de los temas, el modo de evaluarlos. En términos generales, implica posicionamientos claros en torno a los objetos y a los sujetos del conocimiento. Una enseñanza que no se centre en el primero y que exalte el componente vincular de la relación pedagógica corre el riesgo de perder aquello que hace de mediación en esa relación: los objetivos en torno al conocimiento. Pero una enseñanza que no considere a los sujetos corre el riesgo de devenir una exposición de saberes propios, de desarrollos de investigaciones personales que caen en el vacío.

En cuanto a los lugares y situaciones de las que se nutren los docentes para formarse como tales, no se registró el pasaje por instituciones formales de formación docente pero sí un grado importante de capitalización en el presente de experiencias culturales y pedagógicas del pasado. Los relatos pusieron de manifiesto la importancia de la experiencia, ese tiempo extendido para probar y ensayar, que los habría llevado a incorporar saberes en estado tácito. Saberes que permiten desplegar una serie de recursos disponibles para enseñar, pero que también podrían llevar a dedicarle poco tiempo a la preparación de las clases.

Las reflexiones sobre la cátedra como lugar de formación permiten afirmar, asimismo, que el proceso de aprendizaje de los docentes universitarios se daría, en gran medida, en el contexto mismo del trabajo, con todas las dificultades y matices inherentes a los vínculos entre sujetos que ocupan diferentes posiciones en una organización jerárquica.

Destacamos también la fuerza asumida por las figuras de referencia en la narración que los profesores hacen de su proceso formativo. Punto que permite rescatar la dimensión diacrónica de la enseñanza y descentrar la mirada del docente de su propia persona para recuperar la incidencia de las "marcas" internalizadas.

Por último, nos detuvimos en uno de los principales desafíos señalados por los profesores de nuestro estudio: “cómo enseñar mejor". Cuestión que, como afirmamos, no puede pensarse por fuera de una pregunta por el modo en que se piensa a los sujetos y de una reflexión sobre los vínculos que se pretende construir con el medio social en el que la universidad se inserta. 
El trabajo realizado nos lleva a pensar en la influencia que la reflexividad sobre la enseñanza universitaria ejerce en los propios profesores. Creemos posible arriesgar, abriendo la posibilidad de futuras investigaciones, que la recuperación de un saber hacer que opera en general de modo tácito, puede incidir en la construcción social de la docencia universitaria. Interesa entonces rescatar el componente de oficio que muchas veces queda invisibilizado o deslegitimado en relación con otras funciones que, en los últimos años, asumieron mayor presencia en nuestras universidades.

\section{REFERENCIAS}

ADORNO, T. Introducción a la Sociología. Madrid: Editora Nacional, 2002.

ALLIAUD, A.; ANTELO, E. Los gajes del oficio. Enseñanza, pedagogía u formación. Buenos Aires: Aique, 2009.

ANIJOVICH, R. Profesores reflexivos para "repensar lo pensado". Suplemento educativo del Diario Clarín, Buenos Aires, 25 marzo 2013. Disponible en: <http:// www.clarin.com/educacion/Profesores-reflexivos-repensar-pensado_0_890311172. html>. Acceso en: 18 nov. 2013.

ANTELO, E. ¿A qué llamamos enseñar? En: ALLIAUD, A.; ANTELO, E. (Org.). Los gajes del oficio. Enseñanza, pedagogía u formación. Buenos Aires: Aique, 2009. p. 19-37.

ALLIAUD, A.; ANTELO, E. Los gajes del oficio. Enseñanza, pedagogía u formación. Buenos Aires: Aique, 2009.

BARTHES, R. Fragmentos de un discurso amoroso. México: Siglo XXI, 1982.

BECHER, T. Tribus y territorios académicos. La indagación intelectual y las culturas de las disciplinas. Barcelona: Gedisa, 2001.

BERTAUX, D. Los relatos de vida. Perspectiva etnosociológica. Barcelona: Edicions Bellaterra, 2005.

BOLÍVAR, A.; DOMINGO, J.; FERNÁNDEZ CRUZ, M. La investigación biográfico-narrativa en educación. Madrid: La Mmuralla, 2001.

BOLÍVAR, A. Las historias de vida del profesorado. Voces y contextos. Revista Mexicana de Investigación Educativa, México, v. 19, n. 62, p. 711-734, jul./sept. 2014. 
BOURDIEU, P. Intelectuales, política y poder. Buenos Aires: Eudeba, 2006.

BOURDIEU, P.; PASSERON, J. C. Los herederos. Los estudiantes y la cultura. Buenos Aires: Siglo XXI, 2003.

BUCHBINDER, P.; MARQUINA, M. Masividad, hetergeneidad y fragmetnación. El sistema universitario argentino 1983-2008. Buenos Aires: Universidad Nacional de General Sarmiento, 2008.

CARLI, S. El estudiante universitario. Hacia una historia del presente de la educación pública. Buenos Aires: Siglo Veintiuno, 2012.

CARLI, S. La cuestión universitaria en la Argentina (2006-2011): debates, dilemas e hipótesis históricas. Revista Sociedad, Buenos Aires, v. 29-30, p. 105-125, 2011.

CHARLOT, B. La relación con el saber. Elementos para una teoría. Buenos Aires: Libros del Zorzal, 2008.

CHIROLEU, A. Las peculiaridades disciplinarias en la construcción de la carrera académica. Revista Perfiles Educativos, México, n. 99, p. 28-46, 2003.

CLARK, B. El sistema de educación superior. México: Nueva Imagen - UNAM, 1983.

CORNU, L. Transmisión e institución del sujeto. Transmisión simbólica, sucesión, finitud. En: FRIGERIO, G.; DIKER, G. (Comp.). La transmisión en las sociedades, las instituciones y los sujetos. Un concepto de la educación en acción. Buenos Aires: Noveduc-CEM, 2004. p. 27-38.

COULON, A. A condição de estudante. A entrada na vida universitária. Salvador: Ed. EDUFBA, 2008.

DELORY-MOMBERGER, C. Experiencia y formación. Biografización, biograficidad y heterobiografía. Revista Mexicana de Investigación Educativa, v. 19, n. 62, p. $695-710,2014$.

DENZIN, N. Deconstructing the biographical method. Conferencia presentada en la reunión anual de the American Educational Research Association. Chicago, 09 abr. 1991.

DUBET, F. El declive de la institución. Profesiones, sujetos e individuos en la Modernidad. Barcelona: Gedisa, 2006. 
EMILIOZZI, S. Políticas en ciencia y tecnología, definición de áreas prioritarias y universidad en Argentina. Revista Sociedad, Buenos Aires, v. 29-30, p. 149-168, 2011.

EZCURRA, A. M. Igualdad en la educación superior. Un desafío mundial. Provincia de Buenos Aires: IEC-UNGS, 2011.

FREUD, S. Sobre la psicología del colegial. En: Obras completas Tomo 2. Traducción de López Ballesteros y De Torres. Madrid: Biblioteca Nueva, 1996.

GIBBONS, M. Pertinencia de la educación superior en el Siglo XXI. Contribución a la Conferencia Mundial sobre Educación Superior de la UNESCO. The World Bank, Washington, DC, 1998. Disponible en: <www.uv.mx/departamentalizacion/ lecturas/papel/Lectura>. Acceso en: 01 dic. 2011.

GLASER, B. Y; STRAUSS, A. The discovery of grounded theory: strategies for qualitative research. New York: Aldine Publishing Company, 1967.

GLUZ, N. (Ed.). Admisión a la universidad y selectividad social. Cuando la democratización es más que un problema de "ingresos". Los Polvorines: UNGS, 2011.

GOODSON, I. Historias de vida del profesorado. Barcelona: Octaedro, 2004.

GORDON, A. Las políticas de ciencia, tecnología y educación superior en el período 2003-2010 en Argentina. Revista Sociedad, Buenos Aires, v. 29-30, p. 169-193, 2011.

JACKSON, P. Práctica de la enseñanza. Buenos Aires: Amorrortu, 2002.

KNORR CETINA, K. La fabricación del conocimiento. Un ensayo sobre el carácter constructivista y contextual de la ciencia. Buenos Aires: Universidad Nacional de Quilmes, 2005.

MARQUINA, M. El conflicto de la profesión académica: entre la autonomía, la burocratización y la mercantilización. En: RINESI, E.; SOPRANO, G. (Comp.) Facultades Alteradas. Actualidad de EI conflicto de las Facultades, de Immanuel Kant. Buenos Aires: Prometeo, 2007. p. 145-174.

NAISHTAT, F.; GARCÍA RAGGIO, A.; VILLAVICENCIO, S. (Comp.) Filosofías de la universidad y conflicto de racionalidades. Buenos Aires: Colihue, 2001.

NAISHTAT, F. Las dimensiones democráticas de la Universidad y sus razones. En: DELAMATA, G. (Ed.). La Universidad argentina en el cambio de siglo. Buenos Aires: Jorge Baustino-UNSAM, 2004. p. 109-120. 
PARDO, J. L. ¿Y si lo original fuera imitar? Diario El País, 20 oct. 2004. Disponible en: <http://elpais.com/diario/2004/10/20/cultura/1098223205_850215.html>. Acceso en: 01 dic. 2011.

PERRENOUD, P. Saberes de referencia, saberes prácticos en la formación de enseñantes: una oposición discutible. Compte-rendu des travaux du séminaire des formateurs de IUFM. Traducción Gabriela Diker. Grenoble: IUFM, 1994. p. 25-31.

PIERELLA, M. P. La autoridad en la universidad. Vínculos y experiencias entre estudiantes, profesores y saberes. Buenos Aires: Paidós, 2014.

RAMA, C. La Tercera Reforma de la educación superior en América Latina. Buenos Aires: Fondo de Cultura Económica, 2006.

RINESI, E. Ecos de una tradición. Diario Página 12, Buenos Aires, 28 mayo 2013. Disponible en: <http://www.pagina12.com.ar/diario/universidad/10-220959-2013-05-28.html>. Acceso en: 10 marzo 2014.

SAUTU, R. (Comp.) El método biográfico. La reconstrucción de la sociedad a partir del testimonio de los actores. Buenos Aires: Lumiére, 2004.

SENNETT, R. El artesano. Barcelona: Anagrama, 2009.

SERRA, S. ¿Cuánto es “una pizca de sal”? Acerca del juego de la transmisión y las reglas de la pedagogía. En: FRIGERIO, G.; DIKER, G. (Comp.). Educar: saberes alterados. Buenos Aires: Del Estante Editorial. p. 75-82.

SUASNÁBAR, C. et al. Modelos de articulación académica. Cultura e identidad de los docentes-investigadores de la UNLP. Ponencia presentada en el II Encuentro Nacional "La Universidad como objeto de investigación". Buenos Aires: Centro de Estudios Avanzados UBA, 1997.

UNZUÉ, M. Claroscuros del desarrollo de los posgrados en Argentina. Revista Sociedad, Buenos Aires, v. 29-30, p. 127-148, 2011.

WEBER, M. El político y el científico. Madrid: Alianza, 1972.

Recebido em: 09 de agosto de 2016 Aceito em: 29 de novembro de 2016

Endereço para correspondência: 27 de febrero 210 bis (2000), Rosario, Provincia de Santa Fe, República Argentina; pierella@irice-conicet.gov.ar 
